\title{
Avaliação do design de tesouras de poda por meio da distribuição da pressão de contato nas mãos
}

Evaluation of prunning shears design through contact pressure distribution on hands

SILVA, Danilo Corrêa; Doutor; Universidade da Região de Joinville - Univille

danilo@idemdesign.net

PASCHOARELLI, Luis Carlos; Doutor; Universidade Estadual Paulista - Unesp

paschoarelli@faac.unesp.br

\section{Resumo}

Muitas atividades humanas dependem de instrumentos ou ferramentas manuais. Garantir as qualidades ergonômicas desses dispositivos ainda é um desafio. Nesse sentido, o objetivo desse artigo foi avaliar três diferentes modelos de tesouras de poda por meio da distribuição de pressão de contato nas mãos durante uma atividade simulada de uso. Foi utilizado o sistema Grip Versatek Wireless ${ }^{\circledR}$ para mensuração de pressão de contato em uma atividade na qual os voluntários realizaram cortes em espetos de madeira. Os resultados apontam níveis de pressão na ordem de $90 \mathrm{kPa}$ e diferentes distribuições de pressão atribuídas ao design dos modelos. Conclui-se que uma abordagem sistêmica no design desses dispositivos pode favorecer as condições de uso desses dispositivos.

Palavras Chave: tesouras de poda; design ergonômico; pressão de contato.

\begin{abstract}
Many human activities depend on hand tools. However, ensuring the ergonomic qualities of these devices is still a challenge. In this sense, the aim of this paper was to evaluate three different models of pruning shears by the contact pressure distribution on hands during a simulated activity of use. The Grip Versatek Wireless ${ }^{\circledR}$ measurement system was used during an activity in which the volunteers performed cuts on wooden skewers. The results indicate pressure levels in the order of $90 \mathrm{kPa}$ and different pressure distributions caused by the design of the models. It is concluded that a systemic approach in the design of these devices can improve the use conditions of these devices.
\end{abstract}

Keywords: wheelchair; ergonomic design; contact pressure. 


\section{Introdução}

Muitas das atividades humanas, sejam profissionais ou domésticas, requerem o uso de instrumentos ou ferramentas manuais. $\mathrm{O}$ uso de um instrumento adequado auxilia a expandir $\mathrm{O}$ alcance das mãos, multiplicar a capacidade de força e aumentar a precisão e eficiência de uma atividade (TICHAUER; GAGE, 1977). Todas essas interfaces devem ser projetadas para operar com facilidade, rapidez e segurança. No entanto, desenvolver ou adequar esses itens aos utilizadores, garantindo níveis mínimos de usabilidade e conforto, ainda é um desafio. Há atualmente muitos dispositivos que não são adequados para grande parte dos consumidores/usuários (CAMPOS, 2014; GALLEY; EDWARD; HAINES, 2005; HERMANSSON, 1999).

Entre esses dispositivos estão as tesouras de poda (tesouras de jardinagem). As tesouras de poda são utilizadas por jardineiros ou por utilizadores domésticos no corte (poda) de ramos e pequenos galhos de vegetação. São constituídas por lâminas afiadas dispostas em mandíbulas metálicas articuladas, com cabos em seus prolongamentos e, comumente, uma mola de retorno (CAMPOS, 2010). O objetivo desse artigo é avaliar três diferentes modelos de tesouras de poda por meio da distribuição de pressão de contato nas mãos durante uma atividade simulada de uso. Para isso, foi utilizado o sistema de mensuração de pressão de contato Grip Versatek Wireless ${ }^{\circledR}$ e elaborada uma atividade na qual os voluntários realizaram cortes em espetos de madeira.

\section{Revisão Teórica}

\subsection{Instrumentos e Ferramentas Manuais}

Os instrumentos e ferramentas manuais variam em função, mecanismos e design, sendo a maioria específica para a tarefa (DABABNEH et al., 2004). A despeito da tendência de mecanização e automação, muitas indústrias ainda utilizam ferramentas e processos manuais. $O$ trabalho com essas ferramentas é associado à vários riscos de saúde, incluindo ferimentos, que muitas vezes são severos, dispendiosos e frequentes (DIANAT; NEDAEl; MOSTASHAR NEZAMI, 2014). O uso frequente ou por longos períodos desses dispositivos é associado ao surgimento de sintomas de distúrbios musculoesqueléticos (AGHAZADEH; MITAL, 1987; ROSSI et al., 2012). Um grande número de fatores ocupacionais, como esforços extremos, movimentos repetitivos, posturas extremas e traumas mecânicos localizados pode levar ao surgimento desses distúrbios (ALDIEN et al., 2005).

Muitos estudos foram realizados para melhorar o design desses dispositivos e para determinar as configurações ótimas de preensão. Entre as características dos objetos, o tamanho, a forma, e o coeficiente de atrito parecem ser cruciais para as capacidades máximas de força (GOISLARD DE MONSABERT et al., 2012). Com isso, o design é preponderante na ergonomia e usabilidade dos instrumentos manuais. Ferramentas ou instrumentos manuais desenvolvidos com preceitos ergonômicos podem reduzir o desconforto, cargas biomecânicas, riscos de ferimentos e ocorrência de sintomas musculoesqueléticos.

O formato da pega ou empunhadura deve ser projetado para otimizar a área de contato entre a mão e a pega para permitir uma melhor distribuição de pressão, levando a um menor desconforto durante o uso (DIANAT; NEDAEI; MOSTASHAR NEZAMI, 2014). Além disso, pegas revestidas com materiais macios distribuem melhor as forças entre os dedos e palma evitando a concentração de cargas em áreas sensíveis, como o centro da palma das mãos. Forças de preensão concentradas no centro da palma representam um fator conhecido de lesões nos tecidos da 
palma, em especial nos tendões flexores dos dedos e no nervo mediano (CHAFFIN; ANDERSSON; MARTIN, 2006).

A atividade realizada com as tesouras de poda pode acarretar em altas forças de preensão, movimentos repetitivos da mão e postura estática dos ombros (HWANG et al., 2011). Além disso, diferenças no design dessas ferramentas podem ser determinantes na distribuição de forças e pressão de contato. Em uma avaliação com tesouras de poda, Hwang et al. (2011) reportaram que as mulheres utilizaram primariamente as falanges distais dos dedos para realizar as tarefas, enquanto os homens utilizaram as falanges médias dos dedos. Os autores atribuem essas diferenças ao dimensionamento inadequado dessas ferramentas para os indivíduos do sexo feminino, os quais possuem mãos de menores dimensões. Portanto, um design adequado dessas ferramentas pode contribuir para uma melhor distribuição de forças e pressão de contato durante a atividade.

\subsection{Pressão de Contato nas Mãos}

A pressão é calculada pela razão entre a força e a área de contato na qual essa força age. A sua unidade padrão de mensuração no Sistema Internacional (SI) é o Pascal (Pa), que corresponde a $1 \mathrm{~N} / \mathrm{m}^{2}$. Comumente, as referências que tratam da pressão de contato entre o corpo humano e um objeto utilizam o múltiplo quilopascal $(\mathrm{kPa})$ para expressar tais valores (HENNIG, 2008). A distribuição de pressão na superfície palmar da mão humana é uma importante variável na análise de interfaces manuais. Mesmo em uma interação com baixas forças, pode-se obter níveis de pressão relativamente altos durante o uso de um produto se houver concentração em uma pequena área. Altas pressões de contato na pele resultam em desconforto, dor e isquemia. Uma preensão palmar produz uma distribuição de pressão desigual, podendo levar a pressões de até 80kPa nas falanges distais dos dedos (HARIH; DOLŠAK, 2014).

A pele é a primeira estrutura anatômica a sofrer as consequências da pressão de contato, uma vez que a pele representa o revestimento externo da maior parte do corpo. Embora a pele suporte cargas frequentes durante as atividades cotidianas sem maiores problemas, quando essas cargas ultrapassam a variação fisiológica aceitável, ocorre uma resposta à esse traumatismo físico, gerando uma lesão (WHITING; ZERNICKE, 2009). Aldien et al. (2005) discutiram os limiares de dor e desconforto de acordo com as regiões da mão. Os autores indicam um limiar de desconforto de $188 \mathrm{kPa}$, enquanto Fransson-Hall e Kilbom (1993) indicaram um valor de $104 \mathrm{kPa}$. As diferenças encontradas nos estudos sobre o assunto indicam que, embora exista uma conexão entre pressão de contato e desconforto, ela ainda não está bem definida (HARIH; DOLŠAK, 2014). Nesse sentido, ainda há demanda por estudos que quantifiquem as pressões de contato entre a mão e diferentes instrumentos manuais, em particular as tesouras de poda. Sejam de uso profissional ou doméstico, essas ferramentas podem requerer esforços e cargas prejudiciais ao utilizador.

\section{Materiais e Métodos}

Esse estudo teve caráter experimental e transversal, com levantamento de dados junto à indivíduos adultos brasileiros. A participação dos sujeitos nas atividades foi voluntária e individual, não havendo nenhum risco ou constrangimento aos participantes. Foram consideradas as diretrizes do Conselho Nacional de Saúde, sob Resolução 466/2012/CNS/MS/CONEP (BRASIL, 2012) e Norma ABERGO de Deontologia ERG BR 1002 (ABERGO, 2003). 
Os métodos de pesquisa incluíram a aprovação por um Comitê de Ética em Pesquisa (CEP aprovado em 11/09/2014 com o parecer número 800.424) e o aceite prévio da participação nos procedimentos por meio de Termo de Consentimento Livre e Esclarecido (TCLE).

\subsection{Materiais, Equipamentos e Configuração da Atividade}

Esse estudo contou com a utilização dos materiais e equipamentos listados no Quadro 1.

Quadro 1 - Materiais e equipamentos para a coleta com os aros de propulsão de cadeiras de rodas

\begin{tabular}{ll}
\hline Material / Equipamento & Função \\
\hline Protocolo de Identificação & Coletar dados pessoais dos voluntários \\
TCLE & Declaração de participação voluntária \\
Protocolo de Lateralidade & Levantamento da lateralidade dos sujeitos \\
Carteira escolar conjugada simples & Preenchimento dos protocolos de pesquisa \\
Balança digital Welmy W-200 & Mensuração da massa corporal dos voluntários \\
Laptop Lenovo T400 & Utilização do software de coleta \\
Luvas de tecido com o sistema Grip Versatek Wireless ${ }^{\circledR}$ & $\begin{array}{l}\text { Avaliação da pressão de contato durante a interação com } \\
\text { os produtos }\end{array}$ \\
Câmera de vídeo SJ 4000 & Utilizada para registrar a atividade em vídeo \\
Tripé & Suporte para a câmera de vídeo \\
Trena metálica & Medição de altura piso - cotovelo \\
Suporte metálico de parede & Suporte para a realização do corte \\
Espetos de madeira “Ninha” 180x3mm & Utilizado para simular os gravetos a serem cortados \\
Três modelos de tesouras de poda (A, B e C) & Modelos de tesouras avaliados \\
\hline
\end{tabular}

Fonte: o autor

As tesouras de poda (jardinagem) foram objeto de estudo de Campos (2014). Na ocasião, a autora avaliou três diferentes modelos quanto a aspectos de percepção, usabilidade e aplicação de forças durante uma atividade simulada de corte. Para o presente estudo foram selecionados esses mesmos modelos, os quais possuem empunhaduras de características distintas.

Segundo a autora, o modelo A se caracteriza por um cabo com formas levemente arredondadas, seção e uma suave curvatura, além de sulcos para encaixe dos dedos no inferior. A estrutura do cabo desse modelo é fabricada em alumínio maciço e revestida com material polimérico, com textura emborrachada e suave ao toque.

O modelo B se caracteriza por um cabo também com formas ovalizadas, mais arredondadas que o anterior. $O$ cabo superior possui uma curvatura suave, não apresenta sulcos para os dedos e se distancia do superior na extremidade da empunhadura. A estrutura do cabo é confeccionada principalmente em polímero de alta resistência, rígido e com textura levemente ranhurada. 
O modelo $\mathrm{C}$ se caracteriza por possuir um cabo superior ovalizado e inferior redondo. Os cabos possuem curvaturas suaves. O cabo inferior também não possui sulcos para encaixe dos dedos e conta com um mecanismo giratório, que diminui o atrito causado pelo movimento de corte. $\mathrm{O}$ cabo é fabricado principalmente de polímero de alta resistência, rígido e liso.

Todos os modelos de tesouras analisados são adequados apenas para uso com a mão direita. Isso se deve ao posicionamento das lâminas de corte, e também ao mecanismo giratório do cabo inferior do modelo C. A Figura 1 exibe o detalhamento técnico dos modelos avaliados.

Figura 1 - Modelos de tesouras de poda A, B e C respectivamente
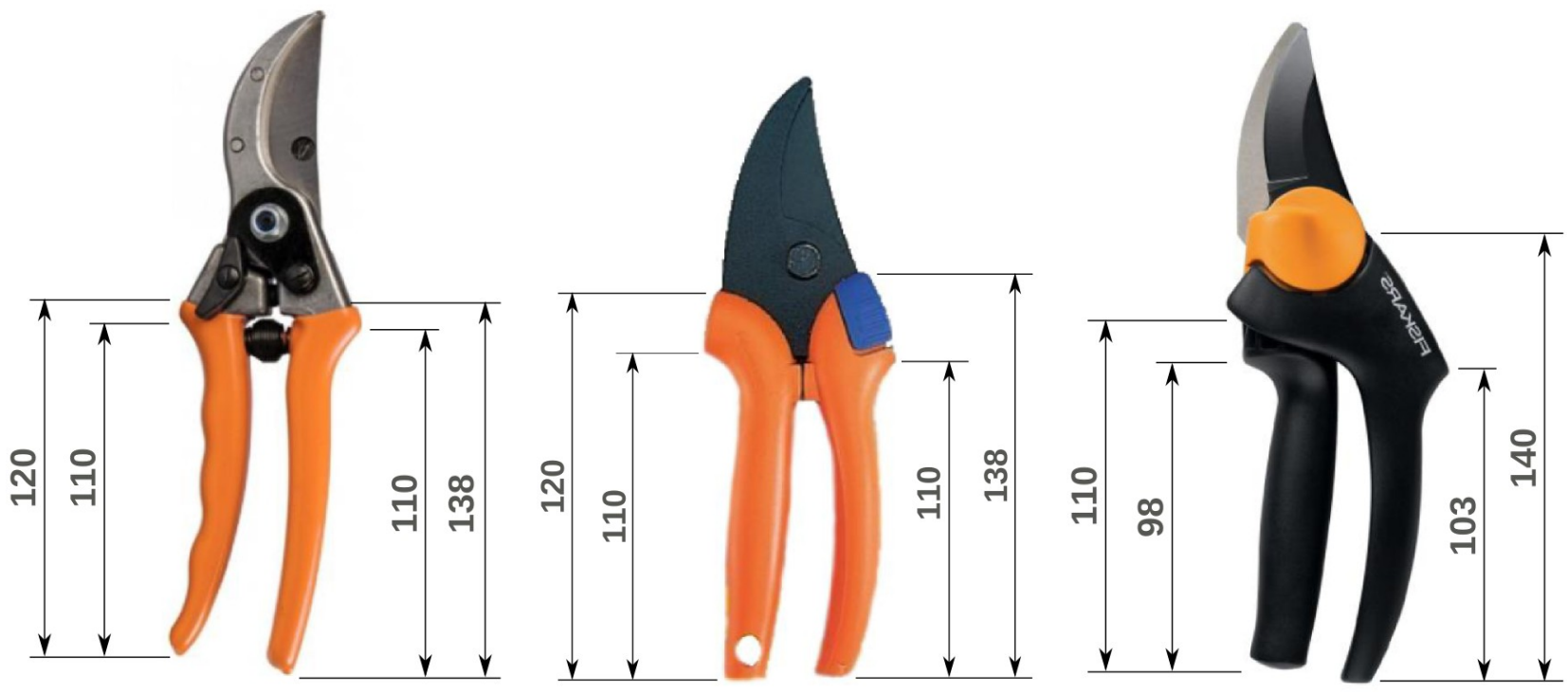

Fonte: o autor

Para avaliar os modelos, Campos (2014) criou um dispositivo de madeira para simular um pequeno arbusto, no qual foram fixados vinte palitos de madeira que, posteriormente, foram cortados com as tesouras.

No presente estudo, optou-se por utilizar um suporte vertical de parede para afixar um único espeto de madeira (pinus), no qual foram marcadas as distâncias de vinte, quarenta e sessenta milímetros a partir da extremidade.

Com isso, a atividade consistiu em efetuar três cortes consecutivos da direita para a esquerda, a cada $20 \mathrm{~mm}$ do espeto. A Figura 2 exibe o esquema de marcação e suporte dos espetos utilizados.

Figura 2 - Marcação para corte nos espetos de madeira

180

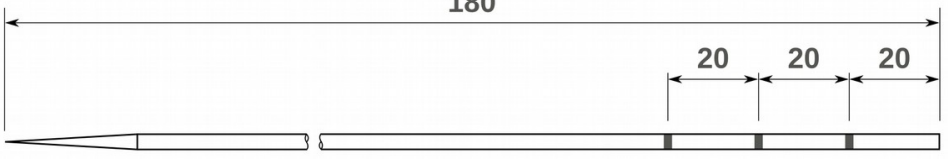

Fonte: o autor 


\subsection{Sujeitos}

Foram considerados inaptos os sujeitos que relataram algum sintoma constante ou lesão musculoesquelética até doze meses antes das atividades. Considerando que os modelos avaliados são específicos para uso com a mão direita, foram considerados inaptos também os sujeitos ambidestros ou canhotos, segundo o protocolo de lateralidade de Oldfield (1971). Houve a participação de 31 (trinta e um) sujeitos aptos, todos com idades entre 18 e 29 anos, e do sexo masculino. Esse recorte foi necessário principalmente devido ao tamanho único das luvas com sensores. A idade média dos participantes foi de 20,5 (d.p. 2,3 anos) e massa corporal média de $72,0 \mathrm{~kg}$ (d.p. 11,6kg). O coeficiente de lateralidade médio foi de 74,8 (d.p. 17,7). Apenas dez sujeitos relataram ter alguma experiência prévia no uso de tesouras de poda.

\subsection{Procedimentos}

Os sujeitos foram abordados individualmente e convidados a participar da coleta. Após o preenchimento dos protocolos de pesquisa, ocorreu uma demonstração de uso do produto, com explicação dos procedimentos, que consistiram em efetuar três cortes consecutivos em um espeto de madeira após um comando verbal, mantendo a postura ereta e o punho em posição neutra. Após a demonstração, os sujeitos calçaram a luva para a mão direita e se posicionaram frente ao suporte em posição ereta e com o cotovelo flexionado em ângulo reto.

A dimensão piso-cotovelo foi tomada para regulagem da altura do suporte metálico. A essa altura foi somado $150 \mathrm{~mm}$, de modo que mesmo segurando a tesoura o sujeito mantivesse o cotovelo a 90 o ou não efetuasse desvio lateral de punho (radial ou ulnar). A ordem de uso dos modelos foi aleatória. Foi então acionada a gravação do vídeo e o software de coleta para início das atividades. $\mathrm{O}$ sujeito efetuou os três cortes consecutiva e ininterruptamente. Houve então a parada da gravação e da coleta de dados em software. A Figura 3 exibe um sujeito executando as atividades com as tesouras e imagens da sequência de vídeo da coleta.

Figura 3 - Sujeito executando a atividade com uma tesoura de poda.
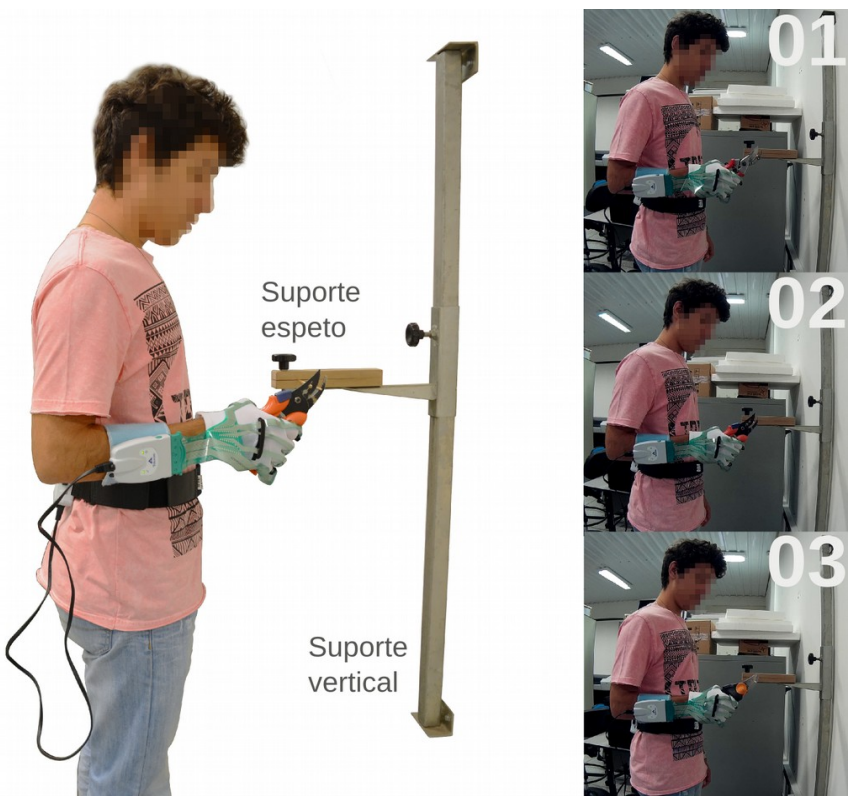

Fonte: o autor 


\subsection{Análises}

Os dados relativos à pressão foram exportados via software segundo cada modelo de tesoura e cada região sensível do sistema. Em seguida, foram importados em planilhas eletrônicas (Libre Office Calc 4) para filtragem e análise. A atividade de corte gerou três picos de pressão. Esses picos foram identificados e foram calculadas suas médias. Seguiu-se a estatística descritiva e testes quanto aos pressupostos de normalidade (Teste de Shapiro-Wilk) e homogeneidade (Teste de Levene). Nos conjuntos de dados onde foram verificadas ambas normalidade e homogeneidade foi aplicada a ANOVA unidirecional e pos hoc de Tukey HSD. Caso contrário foi utilizado o teste de Friedman e pos hoc com o teste de Wilcoxon. Todos os testes estatísticos foram realizados com auxílio do software IBM SPSS 22.

\section{Resultados}

As análises comparativas entre os níveis de pressão em cada modelo revelaram diferenças significativas em doze das dezessete regiões analisadas. O Quadro 1 exibe os testes estatísticos aplicados e os resultados das comparações entre os três modelos de tesouras para cada região analisada.

Quadro 1 - Comparações estatísticas entre modelos para cada região da mão direita.

\begin{tabular}{|c|c|c|}
\hline Região & Teste & Resultado \\
\hline Ppol & Friedman & $\chi^{2}(2)=16,377, p=0,000$ \\
\hline Dpol & Friedman & $\chi^{2}(2)=16,024, p=0,000$ \\
\hline Pind & Friedman & $\chi^{2}(2)=28,667, p=0,000$ \\
\hline Mind & Friedman & $\chi^{2}(2)=8,309, p=0,016$ \\
\hline Dind & Friedman & $\chi^{2}(2)=13,000, p=0,002$ \\
\hline Pmed & Friedman & $\chi^{2}(2)=3,744, p=0,154$ \\
\hline Mmed & ANOVA & $F(2,90)=16,293, p=0,000$ \\
\hline Dmed & Friedman & $\chi^{2}(2)=29,235, p=0,000$ \\
\hline Pane & Friedman & $\chi^{2}(2)=5,131, p=0,077$ \\
\hline Mane & Friedman & $\chi^{2}(2)=28,231, p=0,000$ \\
\hline Dane & Friedman & $\chi^{2}(2)=32,579, p=0,000$ \\
\hline Pmin & Friedman & $\chi^{2}(2)=1,755, p=0,416$ \\
\hline Mmin & Friedman & $\chi^{2}(2)=5,293, p=0,071$ \\
\hline Dmin & Friedman & $\chi^{2}(2)=8,990, p=0,011$ \\
\hline Mc & Friedman & $\chi^{2}(2)=8,051, p=0,018$ \\
\hline Ten & Friedman & $x^{2}(2)=43,626, p=0,000$ \\
\hline Hip & Friedman & $\chi^{2}(2)=4,750, p=0,093$ \\
\hline
\end{tabular}

Fonte: o autor. 
As análises comparativas entre os níveis de pressão em cada modelo revelaram diferenças significativas em doze das dezessete regiões analisadas. Os valores obtidos para os níveis de pressão de contato em cada região da mão analisada em cada modelo de tesoura podem ser visualizados na Figura 4. As comparações cujos valores de pressão de contato foram significativamente maiores são indicadas pela letra do modelo.

Figura 4 - Pressão de contato por área da mão direita em cada modelo de tesoura de poda.
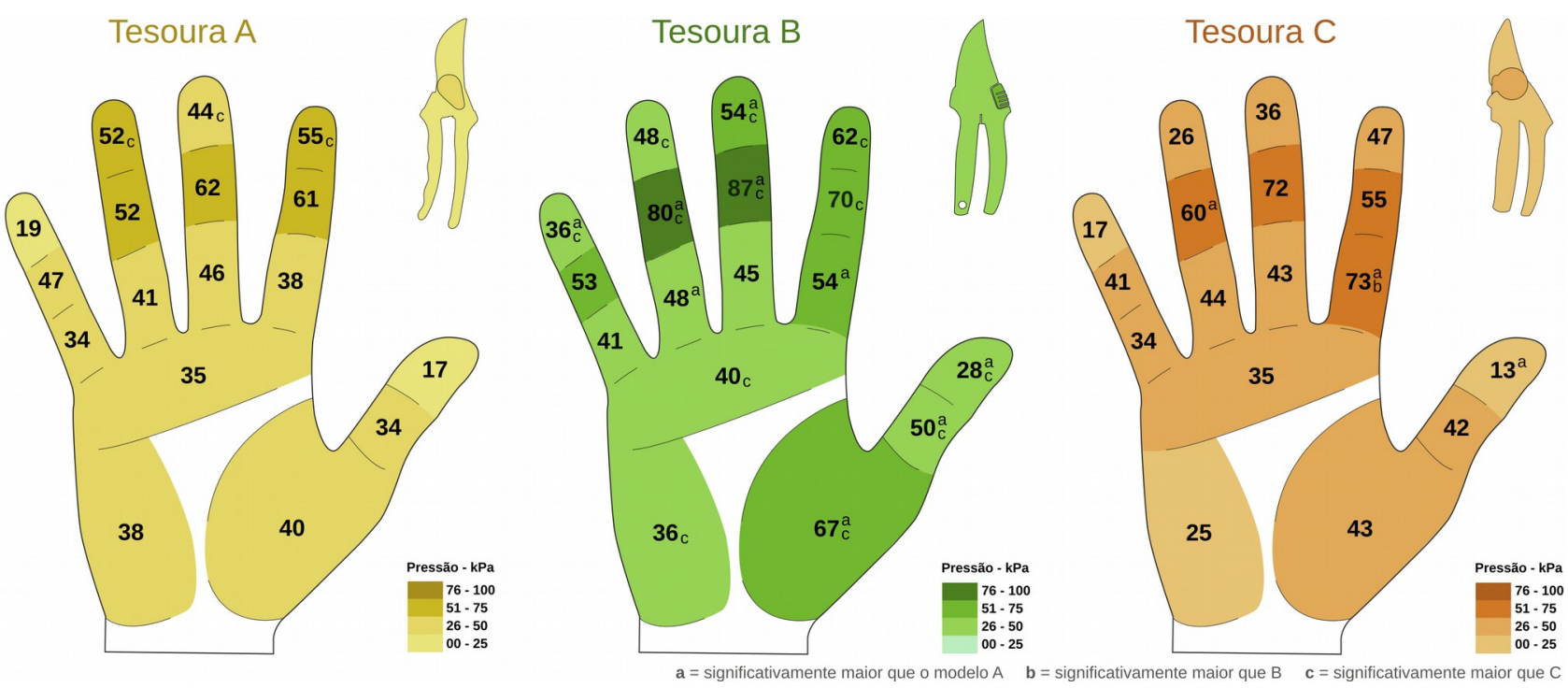

Fonte: o autor.

Como visível na Figura 4, a tesoura A exibiu valores de pressão de contato abaixo das demais em seis regiões analisadas, embora essas diferenças não sejam significativas em todas as ocasiões. As análises pos hoc revelaram que níveis de pressão significativamente maiores ocorreram apenas em relação à tesoura $C$, nas regiões distais dos dedos indicador (Wilcoxon, $Z=-$ 2,242, $p=0,025$ ), médio (Wilcoxon, $Z=-3,289, p=0,001$ ) e anelar (Wilcoxon, $Z-4,625, p=0,000$ ).

A tesoura B exibiu os maiores níveis de pressão em quase todas as regiões da mão. Para o dedo polegar houve registro de pressão significativamente maior na região proximal do polegar, em relação à tesoura $A$ (Wilcoxon, $Z=-3,774, p=0,000$ ) e também à tesoura $C$ (Wilcoxon, $Z=-3,178$, $p=0,001)$. Para o dedo indicador houve maior pressão em relação à tesoura $A$ na região proximal (Wilcoxon, $Z=-2,739, p=0,006$ ) e à tesoura $C$ na região medial (Wilcoxon, $Z=-2,797, p=0,005$ ) e distal (Wilcoxon, $Z=-3,085, p=0,002$ ).

Para o dedo médio, houve registros de maiores níveis de pressão de contato nas regiões medial em relação a $A(T u k e y ~ H S D, p=0,000)$ e $C$ (Tukey HSD, $p=0,003$ ) e também na região distal, em relação a $A$ (Wilcoxon, $Z=-3,034, p=0,002$ ) e C (Wilcoxon, $Z=-4,433, p=0,000$ ).

Para o dedo anelar esse comportamento foi observado na região proximal em relação a $A$ (Wilcoxon, $Z=-2,674, p=0,007$ ) e medial em relação a $A$ (Wilcoxon, $Z=-4,083, p=0,000$ ) e $C$ (Wilcoxon, $Z=-4,124, p=0,000$ ). Na região distal do dedo anelar houve maior pressão apenas em relação a C (Wilcoxon, $Z=-4,236, p=0,000$ ). 
No dedo mínimo houve maiores registros apenas na região distal em relação à $A$ (Wilcoxon, $Z=-2,591, p=0,010$ ) e $C$ (Wilcoxon, $Z=-2,934, p=0,003$ ). A região metacarpal também se mostrou com maiores níveis de pressão em relação à $C$ (Wilcoxon, $Z=-2,016, p=0,044)$. Esse comportamento também foi observado na região tênar em relação a $A$ (Wilcoxon, $Z=-4,860, p=0,000$ ) e $C$ (Wilcoxon, $\mathrm{Z}=-4,762, \mathrm{p}=0,000$ ). Finalmente, para a região hipotênar em relação à $C$ (Wilcoxon, $Z=-2,221$, $p=0,026)$ também houve maior pressão de contato.

Por fim, de acordo com a Figura 4, a tesoura C exibiu o maior nível de pressão apenas na região proximal do indicador. As comparações estatísticas revelam que a tesoura $C$ exibiu níveis de pressão significativamente maiores do que a tesoura $A$ na região proximal do dedo polegar (Wilcoxon, $Z=-2,479, p=0,013$ ). O mesmo foi observado na região proximal do indicador quando comparada à tesoura $A$ (Wilcoxon, $Z=-4,247, p=0,000$ ) e à tesoura $B$ (Wilcoxon, $Z=-3,857, p=0,000$ ). Esse comportamento também foi observado em relação à tesoura $A$ na região medial do dedo anelar (Wilcoxon, $\mathrm{Z}=-2,160, \mathrm{p}=0,031$ ).

\section{Discussão e Conclusões}

A pressão de contato é uma variável importante no design de produtos. Porém, os meios para se quantificá-la no uso de instrumentos manuais ainda são poucos. Nesse artigo foi utilizado o sistema de mapeamento de pressão Grip Versatek Wireless ${ }^{\circledR}$, o qual foi acoplado em luvas de tecido para facilitar o correto posicionamento dos sensores e facilitar o processo de uso. Esse sistema tem se mostrado robusto e capaz de fornecer resultados concisos.

Durante as atividades, foi possível identificar pressões próximas a $90 \mathrm{kPa}$. Valores de pressão nessa ordem estão próximos ao limiar de desconforto relatado por Fransson-Hall e Kilbom (1993), que é de $104 \mathrm{kPa}$ e cerca da metade do exposto por Aldien et al. (2005), que é de $188 \mathrm{kPa}$ para os dedos e 100kPa para a palma da mão. Os elevados níveis de pressão são indicativos de interface com área reduzida, na qual não foi explorado o uso de materiais macios, como sugerido por Hwang et al. (2011) e lida (2005). Todos os modelos possuíam pouco ou nenhum acolchoamento nas pegas. Nesse sentido, o fato de o modelo $A$ ser comparável ao modelo $C$ (o qual tem um design premiado internacionalmente) pode ser devido à sua maior área de pega e material com textura emborrachada e suave ao toque.

Destaca-se aqui também que foi possível notar o mesmo padrão de distribuição de pressão descrito por Hwang et al. (2011), no qual os maiores valores foram observados nas falanges médias dos sujeitos do sexo masculino. Também houve diferenças na distribuição de pressão ocasionadas pelo design da empunhadura (cabo) das tesouras. Os resultados obtidos corroboram os encontrado por Campos (2014), uma vez que a tesoura A e C não diferiram significativamente entre si, e obtiveram os melhores resultados de distribuição de pressão.

Nesse caso, os sulcos e saliências no cabo da tesoura A não parecem ter causado maiores níveis de pressão de contato. Isso pode estar relacionado às características da atividade, na qual a mão direita do sujeito segurou constantemente o cabo da tesoura, situação essa que pode não corresponder à situações de uso diversificadas. De maneira geral, ao se comparar a tesoura A com a C, a primeira exibiu maiores níveis de pressão nas regiões distais dos dedos (indicador, médio e anelar). Já a tesoura $C$ exibiu maiores níveis de pressão de contato nas regiões proximais (indicador) e mediais (anelar). Essa constatação pode estar associada às características dos cabos desses modelos. A primeira, embora com cabo mais longo, tem vão menor que a segunda, enquanto a tesoura $\mathrm{C}$ possui mecanismo giratório para manter a posição dos dedos constante. 
Já a tesoura B apresentou os maiores níveis de pressão. Essa diferença foi significativa para muitas das regiões da mão, principalmente nos dedos indicador, médio, anelar e para a palma da mão. Assim, os experimentos realizados permitem identificar diferenças na distribuição de pressão relacionadas ao design da interface. A ocorrência de níveis elevados de pressão é indicativa da concentração de pressão em estruturas anatômicas específicas da mão.

O design da tesoura $C$ parece se utilizar de preceitos ergonômicos, sendo premiado por suas inovações. Porém, na abordagem realizada por esse estudo, obteve-se resultados similares com um cabo mais longo e material emborrachado (modelo A). Esse é um aspecto essencial ao design dessas ferramentas, visto que possivelmente o investimento na engenharia e no design do modelo $C$ foi consideravelmente maior. Disso conclui-se que a falta de uma abordagem sistêmica no design de interfaces manuais pode comprometer os resultados desses projetos e, consequentemente, afetar empresas e consumidores.

\section{Agradecimentos}

Esse estudo contou com o apoio da Fundação de Amparo à Pesquisa do Estado de São Paulo (FAPESP - Processo 2013/24629-5) e Coordenação para o Aperfeiçoamento de Pessoal de Nível Superior (CAPES) e co Conselho Nacional de Desenvolvimento Científico e Tecnológico (CNPq - Processo 484153/ 2011-0).

\section{Referências}

ABERGO. Norma ERG BR 1002 - Código de Deontologia do Ergonomista CertificadoOuro PretoAssociação Brasileira de Ergonomia, , 2003. Disponível em: <http://www.abergo.org.br/arquivos/normas_ergbr/norma_erg_br_1002_deontologia.pdf>

AGHAZADEH, F.; MITAL, A. Injuries due to handtools. Results of a questionnaire. Applied Ergonomics, v. 18, n. 4, p. 273-278, dez. 1987.

ALDIEN, Y. et al. Contact pressure distribution at hand-handle interface: role of hand forces and handle size. International Journal of Industrial Ergonomics, v. 35, n. 3, p. 267-286, mar. 2005.

BRASIL. Resolução 466BrasíliaPublicada no DOU n 12 - Quinta-feira, 13 de junho de 2013 - Seção 1 - Página 59, 2012. Disponível em: <http://conselho.saude.gov.br/resolucoes/2012/Reso466.pdf>

CAMPOS, L. F. A. Avaliação de forças manuais em atividades simuladas com indivíduos adultos brasileiros de diferentes gêneros e faixas etárias: aspectos do design ergonômico. Bauru: Universidade Estadual paulista "Júlio de Mesquita Filho", 2010.

CAMPOS, L. F. DE A. Usabilidade, percepção estética e força de preensão manual: Influência do design ergonômico de instrumentos manuais - um estudo com tesouras de poda. [s.l.] Universidade Estadual Paulista "Júlio de Mesquita Filho", 2014.

CHAFFIN, D. B.; ANDERSSON, G. B. J.; MARTIN, B. J. Occupational Biomechanics. 4. ed. Hoboken, New Jersey: John Wiley \& Sons, Inc., 2006.

DABABNEH, A. et al. Ergonomics. A checklist for the ergonomic evaluation of nonpowered hand tools. Journal of occupational and environmental hygiene, v. 1, n. 12, p. D135-45, dez. 2004. 
DIANAT, I.; NEDAEI, M.; MOSTASHAR NEZAMI, M. A. The effects of tool handle shape on hand performance, usability and discomfort using masons' trowels. International Journal of Industrial Ergonomics, dez. 2014.

FRANSSON-HALL, C.; KILBOM, A. Sensitivity of the hand to surface pressure. Applied ergonomics, v. 24, n. 3, p. 181-9, jun. 1993.

GALLEY, M.; EDWARD, E.; HAINES, V. Packaging: a box of delights or a can of worms? The contribution of ergonomics to the usability, safety and semantics of packaging. FaraPack Briefing 2005: New Technologies for Innovative Packaging. Anais...Loughborough: 2005Disponível em: <http://hdl.handle.net/2134/3105>

GOISLARD DE MONSABERT, B. et al. Quantification of hand and forearm muscle forces during a maximal power grip task. Medicine and science in sports and exercise, v. 44, n. 10, p. 1906-16, out. 2012.

HARIH, G.; DOLŠAK, B. Comparison of subjective comfort ratings between anatomically shaped and cylindrical handles. Applied Ergonomics, v. 45, n. 4, p. 943-954, jul. 2014.

HENNIG, E. M. Measurement of pressure distribution. In: Routledge handbook of biomechanics and human movement science. 1. ed. New York: Routledge, 2008. p. 143-155.

HERMANSSON, A. Openability of Retail Packages. Packaging Technology and Science, v. 223, n. July, p. 219-223, 1999.

HWANG, J. et al. Design and Assessment of Ergonomics of Hand-Powered Pruning Shears Based On Gender-Specific Operating Strategy. Proceedings of the Human Factors and Ergonomics Society Annual Meeting, v. 55, n. 1, p. 1671-1675, 1 set. 2011.

IIDA, I. Ergonomia: projeto e produção. 2. ed. São Paulo: Editora Edgard Blücher, 2005. v. 1

OLDFIELD, R. C. The assessment and analysis of handedness: the Edinburgh inventory. Neuropsychologia, v. 9, n. 1, p. 97-113, mar. 1971.

ROSSI, J. et al. Characterisation of forces exerted by the entire hand during the power grip: effect of the handle diameter. Ergonomics, v. 55, n. 6, p. 682-92, jun. 2012.

TICHAUER, E. R.; GAGE, H. Ergonomic principles basic to hand tool design. American Industrial Hygiene Association Journal, v. 38, n. 11, p. 622-634, 1977.

WHITING, W. C.; ZERNICKE, R. F. Biomecânica funcional e das lesões musculoesqueléticas. 2. ed. Rio de Janeiro: Guanabara Koogan, 2009. 